\title{
Impact of Male Patient Information on Quality of Urine Examination (PIQUE Study)
}

\author{
Philipp J.Spachmann ${ }^{\mathrm{a}}$ Cathrin Rupp ${ }^{\mathrm{a}}$ Hans-Martin Fritsche ${ }^{\mathrm{a}, \mathrm{b}}$ Stefan Denzinger ${ }^{\mathrm{a}}$ \\ Maximilian Burger ${ }^{\mathrm{a}} \quad$ Johannes Breyer $^{\mathrm{a}} \quad$ Wolfgang Otto $^{\mathrm{a}}$ Marco J. Schnabel ${ }^{\mathrm{a}}$ \\ aDepartment of Urology, University of Regensburg, Caritas St. Josef Medical Center, Regensburg, Germany; \\ bepartment of Urology, Dr. Lubos-Clinics, Munich, Germany
}

\section{Keywords}

Urine culture $\cdot$ Midstream urine $\cdot$ Urinary tract infection .

Urine culture contamination · Antibiotic stewardship

\section{Abstract}

Background: Urine examination has relevance for treatment, and reliability of positive urine culture (UC) is of importance. The technique of urine sampling (US), storage, and transportation is important. The objective of this study was to investigate if detailed patient information for the technique of US and hygiene reduces rates of contaminated UC in screened male patients, as this group was not investigated yet. Methods: All patients independently of complaints were enrolled prospectively and consecutively in an outpatient setting in 2 groups - the first group did not receive detailed information and the second group did. We examined 372 consecutive patients in 2017, 190 not receiving (median age 69 years) and 182 receiving information (median age 70 years), with comparable numbers of patients and age. The result of UC and age was imposed. Results: In all,74.2\% of preclarification UC showed a contamination $(n=95)$ and $75.5 \%$ after clarification $(n=83)$, without significant differences $(p=0.827)$. This study is limited by the fact that adher- karger@karger.com www.karger.com/uin

Karger"
(C) 2021 The Author(s)

Published by S. Karger AG, Basel

This is an Open Access article licensed under the Creative Common Attribution-NonCommercial-4.0 International License (CC BY-NC) (http://www.karger.com/Services/OpenAccessLicense), applicable to the online version of the article only. Usage and distribution for commercial purposes requires written permission. ence could not be checked. Conclusions: Similar to studies with females, no difference occurred in rates of contaminated UC, so detailed information regarding the US technique does not decrease rates of contaminated UC and vice versa does not increase the quality of midstream-sampled UC in male patients.

(c) 2021 The Author(s)

Published by S. Karger AG, Basel

\section{Background}

Urine examination is of great relevance for accurate treatment of infectious diseases as well as for perioperative prophylaxis in surgery of the urinary tract. Thus, the reliability of a positive urine culture (UC) is very important. Factors with impact on the results of UC are not only the microbiology and the clinician's interpretation but also the prediagnostic steps like the technique of urine sampling (US), storage of samples, and transportation [1].

The US is the starting point for clinicians and microbiologists: voided midstream urine is the generally recommended and used technique, but often US is done without any information for patients regarding the right

Correspondence to:

Philipp J. Spachmann, philipp.spachmann@ukr.de 
technique. Accordingly, since UC often shows contamination like bacteria of the skin flora, mixed cultures with $>2$ different bacteria, insignificant count of Enterococci, or insignificant count of Gram-negative bacteria, the interpretation of such results is difficult.

In times of emerging multiresistant bacteria, antibiotic stewardship programs emphasize the importance of reliability of UC as antibiotic treatment depends on this factor not only in the situation of dysuria but also before urologic intervention and surgery to the urinary tract. Usage of antibiotics must be performed very restrictively [2-5]. The significant count of bacteria depends on status of discomfort or not, different definitions in the literature, also for different bacteria, and ways of performing urine collection, but mostly counts of $<10,000$ colony forming units (CFU) per milliliter define insignificant urinary infection [6-9]. Though different studies performed in emergency situation of women with complaints showed no significant differences between voided midstream cultures and other techniques regarding contamination, the knowledge of the situation is low or not existing $[10,11]$.

The objective of this study was to investigate if detailed patient information for the technique of US and improved hygiene reduces the rate of contaminated UC over male patients independently of complaints or not. Via this, we wanted to find out if strict performance of an accurate technique improves situation not only in infected situation with complaints but also prior to urological interventions or surgery in the urinary tract.

\section{Methods}

Three hundred and seventy-two patients were enrolled in 6 weeks between the tenth and nineteenth calendar weeks and the twentieth and thirty-first calendar weeks of 2017 who presented for different reasons in the urologic ambulance and were enrolled prospectively and consecutively independent of complaints or not. The second period was 2 weeks longer due to vacation and low number of patient appearance during this time. The sample size itself was not calculated but a result of the defined periods of time. Patients without UC or under 18 years of age were excluded.

The first group did not receive detailed information on how a UC should be collected. The second group received detailed information in the form of an information brochure and in the form of a poster in the toilet where the cultures were collected.

Both groups showed comparable numbers of patients and structure of age. All patients could read and understand the information sheets. Besides the result of UC (negative/positive/type of bacteria and CFU), age was imposed.

Bacterial contamination of UC was defined by the finding of bacteria of skin flora, cultures with $>2$ findings of bacteria, and enterococci and Gram-negative bacteria with an amount of $<10,000$
CFU per milliliter and without further differentiation. Separately, contamination with Candida spp. was investigated.

Data analyses were performed with SPSS $\odot$ by IBM, Armonk, NY, USA. Statistical differences between both groups regarding positive UC, contamination, and detected bacteria and Candida were determined by the $\chi^{2}$ test, and $p$ value $<0.05$ was defined as significant.

Patient information contained detailed information of hygiene techniques: cleaning of hands with soap, using wet hygiene gloves, and cleaning the genital region and the technique of sampling midstream urine. The information was given to patients as a handout before US. Also, information could be read on a poster in the toilet were US was given. In the toilet, the patients could use hygiene cloths.

\section{Results}

Of all 372 consecutive patients, 190 patients did not receive detailed information, and they were between 21 and 91 years old with a median age of 69 years. One hundred and eighty-two patients received information and were between 23 and 89 years old (median age 70 years).

In preclarification cases, a positive UC was found in 128 cases $(67.4 \%)$ and with clarification UC positive in 110 cases $(60.4 \%)$. No significant differences could be observed pre- and with clarification regarding positive UC ( $\chi^{2}$ test, $\left.p=0.164\right)$. Positive UC in preclarification cases showed contamination in $74.2 \%(n=95)$, and contamination of positive UC with clarification could be observed in $75.5 \%(n=83)$; no significant differences could be detected pre- and with clarification regarding contaminated positive UC $(p=0.827)$.

In preclarification, Escherichia coli and Klebsiella pneumoniae were the mostly found bacteria in 7 positive UC (5.5\%). Staphylococcus aureus was found in 4 positive UC (3.1\%); Morganella morganii in 3 (2.3\%); Candida albicans, Klebsiella oxytoca, Enterococcus faecalis, and Streptococcus agalactiae in 2 UC each (1.6\%); and Pseudomonas aeruginosa, Candida parapsilosis, Proteus mirabilis, and Hafnia alvei in one each (0.8\%). With clarification, E. coli again was the mostly found bacterium in $8 \mathrm{UC}$ (7.3\%); S. aureus in 4 UC (3.6\%); K. oxytoca in 3 UC (2.7\%); and P. aeruginosa and K. pneumoniae in 2 cases (1.8\%). Acinetobacter pittii, C. parapsilosis, Citrobacter freundii, Citrobacter koseri, Enterobacter cloacae, E. faecalis, and Enterococcusfaecium were found in 1 UC (0.9\%) each.

Positive UC preclarification was contaminated with bacteria of the skin flora in 47 cases (36.6\%), insignificant Enterococci in 19 (14.8\%), and insignificant Gram-nega- 
Table 1. Differentiation of findings in positive UC preclarification
Table 2. Differentiation of findings in positive UC with clarification

\begin{tabular}{lcr}
\hline Species/group & Frequency & $\%$ \\
\hline Bacteria of skin flora & 47 & 36.6 \\
Mixed culture of different species & 12 & 9.4 \\
Insignificant Enterococci & 19 & 14.7 \\
Insignificant Gram-negative bacteria without further differentiation & 17 & 13.3 \\
Pseudomonas aeruginosa & 1 & 0.8 \\
Staphylococcus aureus & 4 & 3.1 \\
Streptococcus agalactiae & 2 & 1.6 \\
Escherichia coli & 7 & 5.5 \\
Klebsiella pneumoniae & 7 & 5.5 \\
Klebsiella oxytoca & 2 & 1.6 \\
Proteus mirabilis & 1 & 0.8 \\
Morganella morganii & 3 & 2.3 \\
Hafnia alvei & 1 & 0.8 \\
Enterococcus faecalis & 2 & 1.6 \\
Candida albicans & 2 & 1.6 \\
Candida parapsilosis & 1 & 0.8 \\
\hline
\end{tabular}

UC, urine culture.

\begin{tabular}{lcr}
\hline Species/group & \multicolumn{2}{c}{ Frequency } \\
\hline Bacteria of skin flora & 41 & 37.4 \\
Mixed culture of different species & 15 & 13.6 \\
Insignificant Enterococci & 17 & 15.5 \\
Insignificant Gram-negative bacteria without further differentiation & 11 & 10.0 \\
Pseudomonas aeruginosa & 2 & 1.8 \\
Acinetobacter pittii & 1 & 0.9 \\
Staphylococcus aureus & 4 & 3.6 \\
Escherichia coli & 8 & 7.3 \\
Klebsiella pneumoniae & 2 & 1.8 \\
Klebsiella oxytoca & 3 & 2.7 \\
Citrobacter freundii & 1 & 0.9 \\
Citrobacter koseri & 1 & 0.9 \\
Enterobacter cloacae & 1 & 0.9 \\
Enterococcus faecalis & 1 & 0.9 \\
Enterococcus faecium & 1 & 0.9 \\
Candida parapsilosis & 1 & 0.9 \\
\hline
\end{tabular}

UC, urine culture. tive bacteria without further differentiation in 17 (13.3\%). Mixed culture of different species was found in 12 cases $(9.4 \%)$.

With clarification, positive UC was contaminated by bacteria of the skin flora in 41 cases (37.4\%), insignificant Enterococci in 17 (15.5\%), and insignificant Gram-negative bacteria without further differentiation in $11(10 \%)$. Mixed culture of different species appeared in 15 positive UC (13.6\%).

Candida spp. were found in 3 cases pre- and in 1 after clarification, also not showing significant differences $(p=$ $0.257)$. No significant differences could be observed pre- and with clarification regarding positive UC ( $\chi^{2}$ test, $p=$ $0.164)$. Also, no significant differences could be detected pre- and with clarification regarding contaminated positive UC ( $p=0.827)$ (Tables 1,2$)$.

\section{Discussion}

Similar to other studies regarding female patients with complaints, in this study, no differences occurred in rates of positive UC pre- and with clarification $(p=0.164)$ and in rates of contamination between these groups $(p=$ 
0.827). Also, no differences were observed regarding Candida spp.-positive UC ( $p=0.257)$.

This is of high interest, as voided midstream urine is regarded as a technique of US, especially in avoiding a contamination of UC. This study shows no benefit in informing patients in this special technique regarding positive UC and contamination of UC. Supposed anatomic risk factors for contamination such as the bacterial flora of the foreskin and potential reservoirs for bacteria like the prostatic urethra and the prostate itself or the theory of commensal bacteria in the distal urethra were no risk factors for contamination which could be reduced by informing patients about voided midstream urine.

On the other hand, this study is limited by the following points: reading and adherence of patient information could not be checked as sampling is of midstream urine, and the number of used cloths is no proper way to check adherence of information of hygiene. Also, the size of groups is set by duration of investigated duration of time and was not calculated before starting the study. Furthermore, this study observed only the effect of patient information on contamination in urine collection overall, and there was no differentiation between patients with symptoms or without. Clarifying if male patients have a symptomatic urinary tract infection or a contamination anamnesis, including acute cystitis symptom score, is of great relevance.

But all in all, this study shows that information about defined urine collection is no effective way to decrease percentage of contamination of samples and vice versa does not improve the quality of UC in males with and without complaints. This is of great relevance, as the group of male patients was not investigated yet, and it is well known that males are more often in urological treatment than women.

This study recommends that the importance of instruction of collection of voided midstream urine and the technique of hygiene in males must be discussed, as quality of UC in males with complaints or preintervention or surgery of the urinary tract is of imminent importance for the urologist.

Especially in times of increasing numbers of antibiotic resistances, relevance and indication of antibiotic treatment must be discussed, but this is not the only point: as the quality of a midstream urine-sampled UC by a detailed informed patient seems not to be better than from a patient without information and seems not to be of higher reliability, the suggestion might be that contamination of voided US in UC must be accepted. Also, con- tamination must be accepted as a factor that seems not be able to improve via the long-time recommended technique of voided midstream urine.

\section{Conclusions}

Detailed information regarding the US technique does not decrease rates of contaminated UC in the males and vice versa does not increase the quality of midstreamsampled UC in male patients with and without complaints. Necessity of voided midstream US must be reconsidered in male patients as in females, and contamination itself must be accepted as a risk factor which cannot be improved via patient information.

\section{Statement of Ethics}

The study included no animals, and the patients included were no study participants as they just got handed out special detailed information for US as recommended in international guidelines. So, this study was only about a clarification of standards, and patients were no study participants per se, and there was no written informed consent, but patients were informed orally about the clarification of standards.

\section{Conflict of Interest Statement}

The authors declare that none of them has potential conflicts of interest.

\section{Funding Sources}

No funding was obtained.

\section{Author Contributions}

P.J.S. was involved in data management and analysis and manuscript writing. C.R. was involved in data collection. H.M.F. was involved in protocol and project development. S.D. was involved in protocol and project development. M.B. was involved in protocol and project development. J.B. was involved in data analysis and manuscript editing. W.O. was involved in data analysis and manuscript editing. M.J.S. was involved in data analysis and manuscript writing.

\section{Data Availability Statement}

The datasets used and/or analyzed during the current study are available from the corresponding author on reasonable request. 


\section{References}

1 LaRocco MT, Franek J, Leibach EK, Weissfeld AS, Kraft CS, Sautter RL, et al. Effectiveness of preanalytic practices on contamination and diagnostic accuracy of urine cultures: a laboratory medicine best practices systematic review and meta-analysis. Clin Microbiol Rev. 2016;29(1):105-47.

2 Davies J, Davies D. Origins and evolution of antibiotic resistance. Microbiol Mol Biol Rev. 2010;74(3):417-33.

3 Dyar OJ, Huttner B, Schouten J, Pulcini C, Esgap. What is antimicrobial stewardship? Clin Microbiol Infect. 2017;23(11):793-8.

4 German Federal Ministry of Food and Agriculture. DART 2020: Fighting antibiotic resistance for the good of both humans and animals; 2016.
5 Jump RL, Heath B, Crnich CJ, Moehring R, Schmader KE, Olds D, et al. Knowledge, beliefs, and confidence regarding infections and antimicrobial stewardship: a survey of Veterans Affairs providers who care for older adults. Am J Infect Control. 2015;43(3):298300.

6 Garcia R, Spitzer ED. Promoting appropriate urine culture management to improve health care outcomes and the accuracy of catheterassociated urinary tract infections. Am J Infect Control. 2017;45(10):1143-53.

7 Stamm WE, Counts GW, Running KR, Fihn S, Turck M, Holmes KK. Diagnosis of coliform infection in acutely dysuric women. $\mathrm{N}$ Engl J Med. 1982;307(8):463-8.
8 Hooton TM, Roberts PL, Cox ME, Stapleton AE. Voided midstream urine culture and acute cystitis in premenopausal women. N Engl J Med. 2013;369(20):1883-91.

9 Murray PR; American Society for Microbiology. Manual of clinical microbiology. 7th ed. Washington: ASM Press; 1999.

10 Maher PJ, Brown AEC, Gatewood MO. The effect of written posted instructions on collection of clean-catch urine specimens in the emergency department. J Emerg Med. 2017; 52(5):639-44.

11 Pernille H, Lars B, Marjukka M, Volkert S, Anne H. Sampling of urine for diagnosing urinary tract infection in general practice first-void or mid-stream urine? Scand J Prim Health Care. 2019;37(1):113-9. 